\title{
ANTIBIOTIC RESISTANCE AND CHILD MORTALITY IN INDIA
}

\author{
A. TAH AND B. GHOSH* \\ Neucrad Health, Philadelphia, US
}

\begin{abstract}
"Antibiotic Resistance" has become a worldwide threat because the resistant bacteria can rapidly spread anywhere. Each year millions of people develop antibiotic resistant infections, some of which lead to death. As per the recent report of Center for Disease Dynamics, Economics \& Policy (CDDEP), antibiotic resistant infections are spreading in India and killing over 58,000 children every year. Antibiotics save the lives of children with serious illnesses such as pneumonia, meningitis and septicemia. Antibiotics can also be used to treat middle ear infections and skin infections in children.

When antibiotics are overused or not used properly for treating infections in children, it become harder to treat them due to antibiotic resistance. Patient, who has developed antibiotic resistance, has lost its ability to effectively control or kill bacterial growth in the presence of therapeutic levels of an antibiotic.
\end{abstract}

That's why it's so important that children only take antibiotics that have been prescribed by physicians. It is important to complete the entire course of antibiotics. Sometimes your children may feel good after taking medicine for $1 / 2$ days.

There is a huge list of organisms those are responsible for significant clinical impact in children. In 1960s methicillin resistant Staphylococcus aureus (MRSA) was recognized as serious threat for the adult population which was comparatively uncommon in pediatric population until 1990s. Soon after, it became a rising threat for the children too. In 1980s antibiotic resistant Enterococcus sp. brought great difficulty in healthcare settings when the organism started showing resistance to vancomycin. Soon vancomycin resistant Enterococcus became threat to the pediatric population (Medernach et al., 2018; Adams et al., 2016).

${ }^{*}$ Corresponding Author: Founder and Managing Director, Neuroscientist, Biochemist and Global

Consultant (Research \& Development), Philadelphia, US 
It is a fact that antibiotic resistance is a global problem. However, it is also true that we are highly dependent on antibiotics for infectious diseases. If not eradicated, at

\section{REFERENCES}

Adams DJ, Eberly MD, Goudie A and Nylund CM, 2016. Rising vancomycin-resistant Enterococcus infections in hospitalized Children in the United States. Hosp Pediatr, 6(7): 404-411 least it can be reduced to a certain level by taking measures at an individual, institutional and finally at national healthcare level.
Medernach RL and Logan LK, 2018. The growing threat of antibiotic resistance in children. Infect Dis Clin North Am, 32(1): $1-17$ 